\title{
Chikungunya virus: emergence of an arthritic arbovirus in Jeddah, Saudi Arabia
}

R. Hussain, ${ }^{7}$ I. Alomar ${ }^{2}$ and Z.A. Memish ${ }^{3}$

\section{Introduction}

Chikungunya virus, an arthritogenic alpha virus, was first described in Tanzania in a febrile female [1]. The disease is endemic in many African countries as well as in South-East Asia (Indonesia, Thailand, Viet Nam, Myanmar, Cameroon, Philippines and Malaysia) [2]. From 2005-06 outbreaks were also reported in the Indian Ocean islands of Comoros, Mayotte, Madagascar, La Reunion, Mauritius and Seychelles [3-6]. Recently it has been reported in travellers from affected areas returning to European countries including Germany, Belgium, Switzerland, Norway and Italy [7].

We report the first case of autochthonous acute chikungunya infection in Saudi Arabia.

\section{Case report}

On 15 May 2011 a serum sample was received from a private hospital in Jeddah for suspected dengue fever. The 55-year-old housewife had complained of arthralgia and myalgia since 5 May 2011. The arthralgia started in the small joints of the hands and later spread to the entire body. By 12 May 2011, she started to have fever with mild abdominal pain and vomiting along with skin rashes. The joint pains were so severe that she could not walk and was brought to the hospital in a wheelchair.
Her temperature on presentation was $39^{\circ} \mathrm{C}$ with blood pressure of $120 / 80$ $\mathrm{mmHg}$. The haematocrit was $34.5 \%$ with platelet count of $198 \times 10^{9} / \mathrm{L}$.

There was no history of travel outside the country or any contact with animals or birds. Physical examination was noncontributory. Preliminary diagnosis of dengue was made and a blood sample was collected. Serum was separated and sent to the Jeddah Regional Laboratory for virological diagnosis.

\section{Virological analysis}

The serum sample was run for dengue fever but both real-time reverse transcriptase polymerase chain reaction (qRT-PCR) assay and serology were negative. As per Ministry of Health laboratory policy all dengue-negative samples were screened for other relevant viral haemorrhagic fevers of concern in Saudi Arabia, which the time included PCR assay for West Nile fever, Rift Valley fever, chikungunya fever, Crimean-Congo haemorrhagic fever and Alkhurma viruses (TIB MOLBIOL).

Automated extraction of RNA was done using the MagNA Pure Compact RNA isolation kit (Roche Diagnostics). The RNA was transcribed into cDNA with transcriptor first-strand cDNA synthesis kit (Roche Diagnostics). The LightMix qRT-PCR kits for the respective viruses (TIB MOLBIOL) and Light Cycler FastStart DNA Master HybProbe reaction mix (Roche) was used for the qRT-PCR with the Light Cycler 2.0 thermocycler (Roche) for each of the suspected virus. For the data analysis, the colour compensation mode was enabled and the second derivative maximum method was used. The virus data were viewed in channel 640 , and the internal control in channel 705 , quantification modes, for dengue, West Nile, Alkhurma, chikungunya and Rift Valley fever viruses. However the Crimean-Congo data were analysed in channel 530 and internal control in 560, quantification modes.

The chikungunya virus qRT-PCR showed clear amplification signals.

The serum sample was run for complete dengue serology, $\operatorname{IgM}$ and $\operatorname{IgG}$, as well as non-structural protein- 1 antigenNS1 (Panbio Diagnostics). The sample was negative for all parameters.

Antibodies against chikungunya were detected using IgM microcapture enzyme-linked immunosorbent assay (ELISA) and IgG capture ELISA (NovaLisa, NovaTec Immunodiagnostica). IgM was 3.79 NovaTec units (NTU) while IgG was 1.94 NTU. Positive values for both IgM and IgG as recommended by the manufacturer are $>11$ NTU.

Sequencing confirmed the presence of chikungunya virus in the sample. The partial amino acid sequencing in 226 region of the E1 gene was carried out for detection of $A 226 \mathrm{~V}$ mutation with primers developed by TIB 
MOLBIOL (Genbank accession number AF369024).

Follow-up samples were obtained from the patient and 6 of her family members on 23 May 2011 and were run again for haemorrhagic fever panels. The patient had PCR negative for chikungunya, while serology demonstrated significant titres of anti- chikungunya (anti-CHICKV) IgM (31.3 NTU) as well as IgG (27.6 NTU) and this confirmed the diagnosis of chikungunya infection. One of the family member also had anti-CHIKV IgM (31.5 NTU) as well as anti-CHIKV IgG (45.1 NTU), while another had only anti-CHICKV IgG (29.5 NTU) with negative PCR. However, a third family member was dengue positive by PCR, while serology for chikungunya virus and PCR were negative.

\section{Discussion}

The mosquito-borne alphaviruses that are prevalent in the tropics and subtropics and are associated with arthritis in humans are chikungunya, o'nyong-nyong, Mayaro, Ross River, Sindbis and Barmah Forest viruses. All of them cause symptoms of fever, joint pains and rash. The symptoms are of short duration, around 1 week, with complete recovery except in the case of chikungunya infection [8]. Chikungunya viral infection causes an algo-eruptive syndrome with disabling joint pains and recurrent rheumatic manifestations [9-11]. Symptoms may be severe and long lasting and articular destruction has also been described [12]. Persons infected with chikungunya virus for more than a year have also been reported to have greater disabilities, complaining more frequently of arthralgia, myalgia, fatigue, depression and hair loss [6].

We report the first laboratory-confirmed case of autochthonous acute chikungunya infection in Saudi Arabia. The case was diagnosed by PCR followed by sequencing. The partial nucleotide sequence analysis of the E1 genome at 226 locus revealed alanine residues at this position. The isolate was therefore of the wild type and not mutant $A 226 \mathrm{~V}$ with valine residue, which is more adapted to the Aedes albopictus mosquito [13]. This mutation has enhanced chikungunya virus infectivity, replication and transmission by $A$. albopictus without impairing the vectorial capacity of A. egypti [14].

The $A 226 \mathrm{~V}$ mutation was responsible for the 2005-06 epidemic in La Reunion island where it infected $34 \%$ of the population and caused 260 deaths $[15,16]$. Also $A 226 \mathrm{~V}$ has been noted in Italy in imported as well as autochthonous cases [17]. Two autochthonous cases of dengue and chikungunya have also been reported from France [18].
Since the vector, A. albopictus, is present in Jeddah, Saudi Arabia, along with A. egypti [19], the discovery of this particular variant alerts us to the risk of rapid spread of the disease due to improved replication and transmission efficacy [14].

The follow-up samples from the patient confirmed seroconversion in the convalescent sample. The endemic status of dengue in Jeddah, and varying levels of influenza activity in the background, highlight the difficulty of making an appropriate clinical diagnosis of chikungunya virus infection, especially in an autochthonous case. Neighbouring Yemen has reported an outbreak of chikungunya virus with 1000 cases and 75 deaths by end of 2010 [20]. The presumed co-circulation of these viruses should raise concerns about the viral haemorrhagic surveillance activity in Jeddah where heightened vigilance and strong epidemiological and laboratory surveillance will be required. It also points to the fact that chikungunya virus infection should be included in the differential diagnosis of fever and arthritis in Saudi Arabia

\section{Acknowledgements}

We thank Olfert Landt (TIB MOLBI$\mathrm{OL}$ ) and Ute Bottcher (GenXpress) for their technical support in sequencing.

Competing interests: None declared.

\section{References}

1. Ross RW. The Newla epidemic iii The virus: isolation, pathogenic properties and relationship to the epidemic. Journal of Hygiene, 1956, 54:177-191.

2. Paquet $\mathrm{C}$ et al. Chikungunya outbreak in Reunion: epidemiology and surveillance, 2005 to early January 2006. Euro Surveillance, 2006, 11:E060202.

3. Mason PJ, Haddow AJ. An epidemic of virus disease in southern province, Tanganyika territory in 53; an additional note on chikungunya virus isolations and serum antibodies. Transactions of the Royal Society of Tropical Medicine and Hygiene, 1952, 51:238-240.

4. Enserink M. Infectious diseases. Massive outbreak draws fresh attention to little known virus. Science, 2006, 311:1085.
5. Gratz NG. Emerging and resurging vector-borne diseases. Annual Review of Entomology, 1999, 44:51-75.

6. Soumahoro MK et al. Impact of chikungunya virus infection on health status and quality of life a retrospective cohort study. PLoS ONE, 2009, 4:e7800.

7. Editorial team, Pfeffer M, Loescher T. Cases of chikungunya imported into Europe. Eurosurveillance, 2006, 11(11):pii 2922.

8. McGill PE. Viral infections: alpha-viral arthropathy. Bailliere's Clinical Rheumatology, 1995, 9:145-150.

9. Borgherini G et al. Outbreak of chikungunya on Reunion Island: early clinical and laboratory features in 157 adult patients. Clinical Infectious Diseases, 2007, 44:1401-1407. 
10. Lokireddy S, Vemula S. Vadde R. Connective tissue metabolism in chikungunya patients. Virology Journal, 2008, 5:31.

11. Fourie ED, Morrison JG. Rheumatoid arthritic syndrome after chikungunya fever. South African Medical Journal, 1979, 56:130-132.

12. Brighton SW, Simson IW. A destructive arthropathy following chikungunya virus arthritis-a possible association. Clinical Rheumatology, 1984, 3:253-258.

13. Ttsetsarkin KA et al. A Single mutation in chikungunya virus affects vector specificity and epidemic potential. PLoS Pathogens, 2007, 3:e201.

14. Lamballerie $X$ et al. Chikungunya virus adapts to tiger mosquito via evolutionary convergence: a sign of things to come? Virology Journal, 2008, 5:33.

15. Charrel RN, de Lamballerie X, Raoult D. Chikungunya outbreaks: the authors reply. New England Journal of Medicine, 2007, 356:2651-2652.
16. Simon F et al. Chikungunya infection: an emerging rheumatism among travelers returned from Indian Ocean Islands. Report of 47 cases. Medicine; Analytical Reviews of General Medicine, Neurology, Psychiatry, Dermatology, and Pediatries, 2007, 86:123-137.

17. Rezza G et al. Infection with chikungunya virus in Italy: an outbreak in a temperate region. Lancet, 2007, 370:1840-1846.

18. Gould EA et al. First cases of autochthonous dengue fever and chikungunya fever in France: from bad dream to reality! Clinical Microbiology and Infection, 2010, 16:1702-1704.

19. Ghaznawi $\mathrm{HI}$ et al. Surveillance for dengue fever in Jeddah. Eastern Mediterranean Health Journal, 1997, 3:567-570.

20. EpiSouth Weekly Epi Bulletin, No. 155, 3 March 2011-9 March 2011. Network for Communicable Disease Surveillance Control in Southern Europe and Mediterranean Countries [online bulletin] (http://www.episouth.org/download.php?\&id=13242, accessed 18 February 2013). 